\title{
High Isolation Internal Dual-Band Planar Inverted-F Antenna Diversity System with Band-Notched Slots for MIMO Terminals
}

\author{
Ki-Jin Kim, Won-Gyu Lim and Jong-Won Yu \\ Department of Electrical Engineering Korea Advanced Institute of Science and Technology (KAIST), \\ 373-1 Guseong-Dong, Yuseong-Gu, Taejeon, 305-701, Korea
}

\begin{abstract}
Internal dual-band planar inverted-F antenna (PIFA) diversity system for portable devices, operating in the $2.4-\mathrm{GHz}$ band $(2400-2484 \mathrm{MHz})$ and $5.2-\mathrm{GHz}$ band $(5150-$ $5350 \mathrm{MHz})$, is presented. To reduce the mutual coupling and get the high isolation between the two internal dual-band antennas, we proposed the band-notched $\lambda / 4$ slots on the ground plane. The optimized band-notched slots for high isolation, high radiation efficiency and high effective diversity gain are analyzed. The band-notched slots can be used to the small MIMO terminals which require high isolation between antennas.

Index Terms - Antenna isolation, band-notched slots, internal antenna, MIMO, PIFA.
\end{abstract}

\section{INTRODUCTION}

With the rapid spread of internet, broad band communication access such as wireless local area networks (WLAN) has been studied actively during the past decades to increase the quantities of the data and improve the quality of the information. However, signal fading due to multipath propagation is an additional factor that impairs the capacitylimiting problem. The use of multi-element antennas, such as a multiple input multiple output (MIMO) system, is one of the effective ways for improving reliability and increasing the capacity of the wireless channel [1]. The capacity of the wireless channels is affected by the mutual coupling between the antenna elements [2]. So, high isolation between the antenna elements, which is possible to receive uncorrelated signal even though they are closely spaced, is required for MIMO terminals. In portable devices, 2 to 4 antenna elements are considered and the restricted space available for the antennas is an open issue. Recently, many studies on reducing mutual coupling between two antennas - two 2.4-GHz/5.2$\mathrm{GHz}$ dual-band antennas with the reflector[3-4], two 5.2-GHz single antennas with the additional ground plane[5], two 5.2$\mathrm{GHz}$ single antennas with a single $\lambda / 4 \operatorname{slot}[6]$ - have been presented.

In this paper, we present a study on the isolation of two internal dual-band WLAN antennas with the band-notched slots for a PDA phone. In the study, dual-band WLAN antenna, operating in the 2.4-GHz band (2400-2484MHz) and $5.2-\mathrm{GHz}$ band $(5150-5350 \mathrm{MHz})$, is printed PIFA with compact size (see Fig. 1.) and is suitable to be mounted at the top and side edge of the system circuit board as internal antennas.

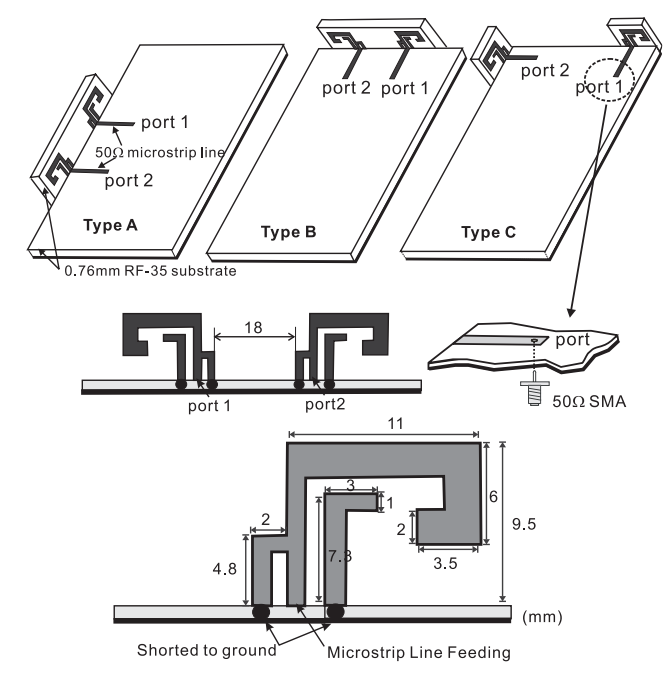

Fig. 1. The geometry and dimensions of the proposed internal planar inverted-F antenna (PIFA) diversity system

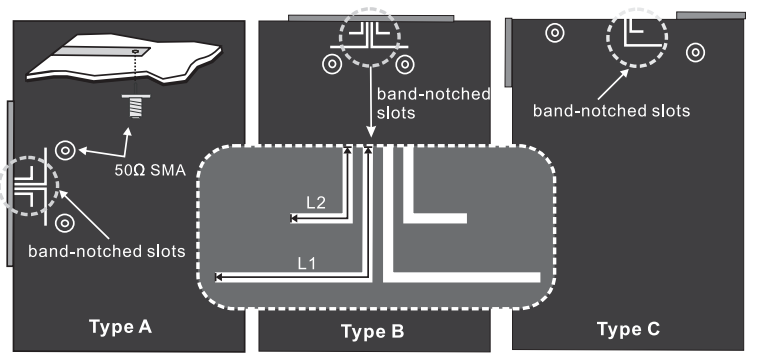

Fig. 2. The geometry and dimensions of the proposed dual bandnotch slots on the ground plane

\section{ANTENNA AND BAND-NotCH SLOT DESIGN}

The geometry and dimensions of the investigated configuration are depicted in Fig. 1. As a basic antenna element for MIMO terminals, the PIFA is considered because the shorting strip of PIFA will function as a reflecting metal plate[6]. The antennas are printed on a 0.76-mm-thick Taconic RF-35 substrate with $\varepsilon_{r}=3.5$ and $\tan \delta=0.0018$. The longer or upper radiating arm controls the resonant mode for 2.4-GHz WLAN operation and is folded downward to achieve a compact configuration. The shorter or lower Lshaped arm, which is enclosed by the longer radiating arm, controls the resonant mode for the 5.2-GHz WLAN operation. 
By adjusting the lengths of arms, the desired lower and upper operating frequencies can b easily obtained. The dual-band WLAN antenna occupies a small area of $9.5 \times 13 \mathrm{~mm}^{2}$ and is mounted at the top or side edge of a system circuit board having a ground plane of 100 -mm length and 70 -mm width in this study, which is a reasonable size for practical PDA phones. Types A and B are for the closely spaced two PIFAs, which are both mounted at the top edge and at the side edge of the printed circuit board(PCB). Type $\mathrm{C}$ is for the orthogonally spaced two PIFAs, which are each mounted at the top and side edge of PCB. Each PIFA is easily fed by using a $50 \Omega$ microstrip line, printed on the front surface of the grounded substrate.

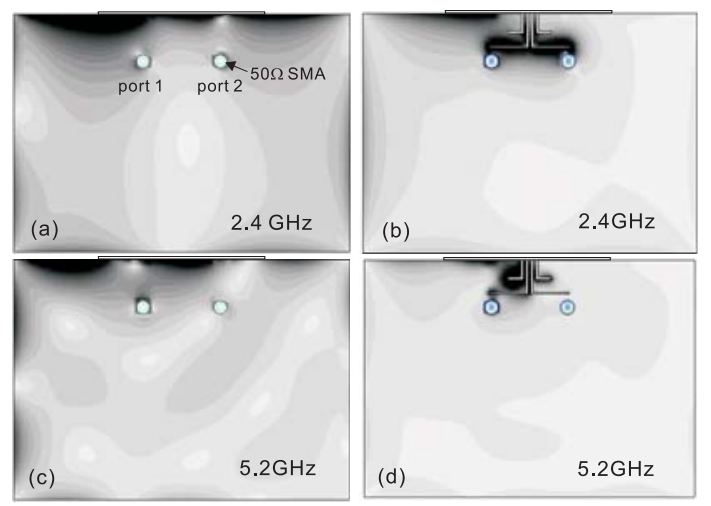

Fig. 3. Normalized surface current on the ground plane (a) without the band-notched slots at $2.4 \mathrm{GHz}$ (b) with the ban-notched slots at $2.4 \mathrm{GHz}$ (c) without the band-notched slots at $5.2 \mathrm{GHz}$ (d) with the band-notched slots at $5.2 \mathrm{GHz}$

As the distance between two PIFAs is decreased, the mutual coupling between two PIFAs is increased and the radiation efficiency of the each antenna is decreased rapidly. To reduce the mutual coupling between two PIFAs, here we introduce the band-notched slots on the ground plane. The geometry and dimensions of the band-notched slots are shown in Fig. 2. For a symmetric radiation pattern, a pair of symmetric dual band-notched slots is etched in the middle of the PIFAs for types A and B. However, an asymmetric dual band-notched slot is etched in the middle of the PIFAs for type $\mathrm{C}$, because the radiation patterns of type $\mathrm{C}$ are asymmetric.

The normalized surface current distributions with/without the band-notched slots at $2.4-\mathrm{GHz}$ and $5.2 \mathrm{GHz}$ are shown in Fig. 3. The mutual coupling between two PIFAs is mainly dependent on the surface current at the edge of the ground plane. The etched feature becomes resonant at the frequency where the length $\boldsymbol{L}_{\mathbf{1}(2)}$ is the quarter wavelength. In Fig. 3(b) and (d), we can see more and stronger current distributions near the bottom edge of a L-shaped slot at the notch frequency. At the frequencies, current is concentrated around the bottom edge of L-shaped slot and is oppositely directed between the interior and exterior of the slot. This causes the ground plane to operate in a transmission line-like mode, which transforms the nearly zero impedance (short circuit) at the bottom of the slot to nearly high impedance (open circuit) at the top of the slot like a $\lambda / 4$ transmission line transformer. This high impedance at the top of the slot leads to the desired high attenuation near the notched frequency.

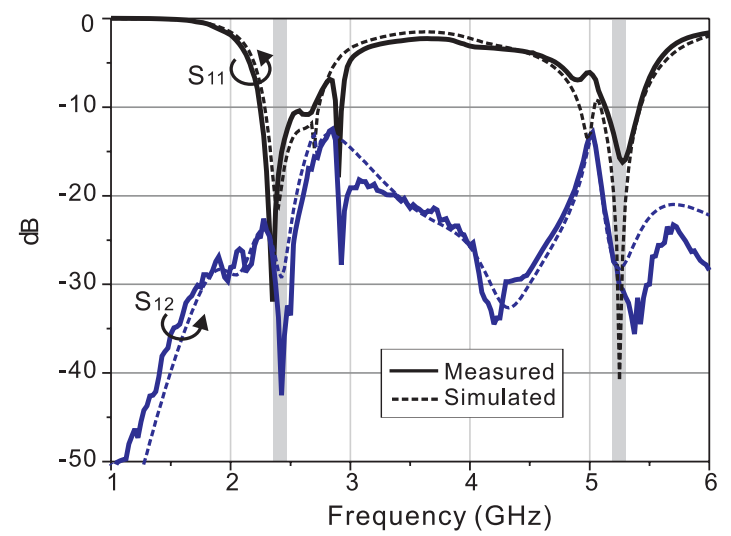

Fig. 4. Measured and simulated S-parameters of the proposed PIFA with band-notched slots for the case of type A
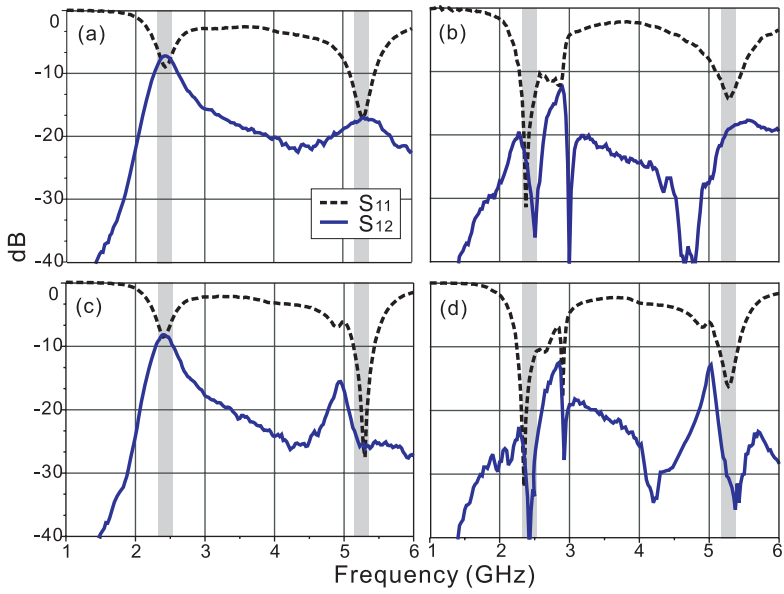

Fig. 5. Measured S-parameters of the PIFA for type A (a) without the band-notched slots (b) with the $2.4 \mathrm{GHz}$ band-notched slot (c) with the $5.2 \mathrm{GHz}$ band-notched slot (d) with the 2.4/5.2GHzdual band-notched slots

\section{EXPERIMENTAL RESULTS AND DISCUSSION}

A plot of the measured and simulated S-parameters against frequency is shown in Fig. 4 for the case of type A and the band-notched slots $L_{1}=21.5 \mathrm{~mm}$ and $L_{2}=10.2 \mathrm{~mm}$. Good agreement is achieved. The simulation employed a timedomain finite integration technique (CST Microwave Studio). The return loss is greater than $10 \mathrm{~dB}$ from 2.23 to $2.70 \mathrm{GHz}$ and from 5.11 to $5.40 \mathrm{GHz}$.

Fig. 5 shows the variations of the s-parameters by inserting band-notched slots on the ground plane. Fig. 5(a) shows the high mutual coupling between the PIFAs without the bandnotched slots. In Fig. 5(b), when we insert the lower bandnotched slot on the ground plane, only lower band mutual 
coupling $\left(\mathrm{S}_{21}\right)$ is suppressed dramatically about $30 \mathrm{~dB}$. Moreover, antenna bandwidth of lower band is substantially increased by decreasing the mutual coupling and by increasing the number of ground current paths. When we insert higher band-notched slot on the ground plane as shown in Fig. 5(c), we can find out that only higher band isolation is improved about 10dB. From the above results, we also know that the mutual coupling between the dual band-notched slots is very little. So, it is possible to design the dual band-notched slots and the measured results are shown in Fig. 5(d). With the dual band-notched slots, the lower and upper band isolations are increased and radiation efficiencies also are improved.

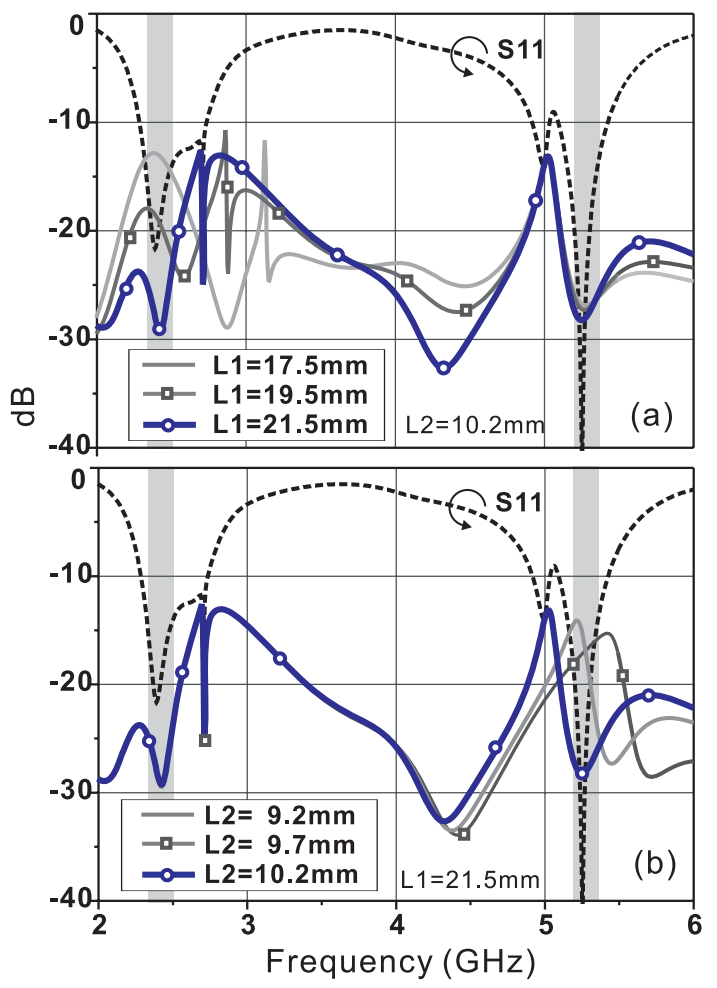

Fig. 6. Isolation $\left(\mathrm{S}_{12}\right)$ for the band-notched slots with various slot lengths $L_{1}$ and $L_{2}$
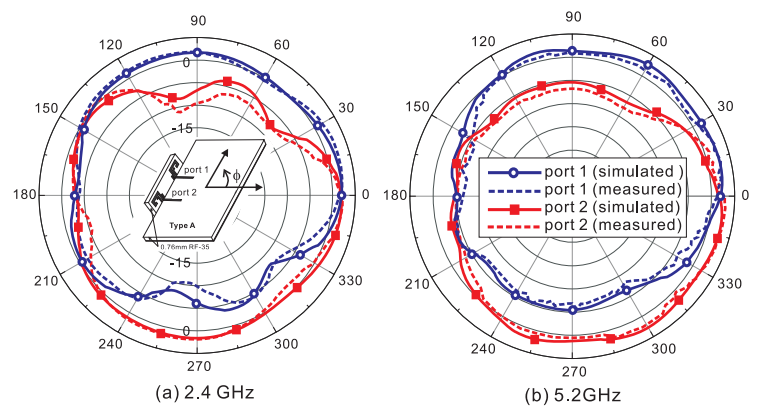

Fig. 7. Measured and simulated antenna gain pattern for the type A with the band-notched slots (a) at $2.4 \mathrm{GHz}$ (b) at $5.2 \mathrm{GHz}$

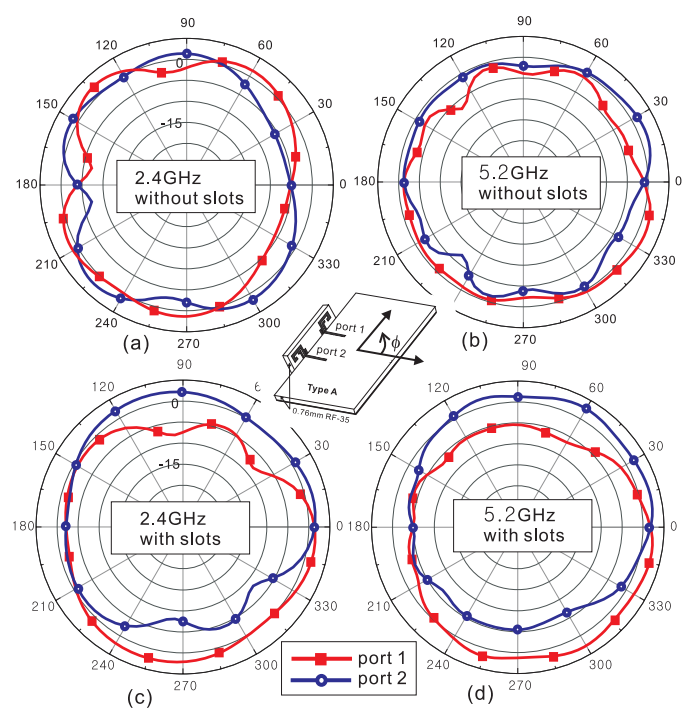

Fig. 8. Antenna gain patterns at the azimuth plane for the type A diversity system (a) at $2.4 \mathrm{GHz}$ without the band-notched slots (b) at $5.2 \mathrm{GHz}$ without the band-notched slots (c) at $2.4 \mathrm{GHz}$ with the bandnotched slots (d) at $5.2 \mathrm{GHz}$ with the band-notched slots

TABLE I

ISOLATION $\left(\mathrm{S}_{21}\right)$ AND RADIATION EFFICIENCY(RE) OF THREE TYPES OF ANTENNA DIVERSITY

\begin{tabular}{|c|l|c|c|c|c|}
\hline \multicolumn{2}{|c|}{} & \multicolumn{2}{c|}{$\begin{array}{c}\text { wlo band- } \\
\text { notched slots }\end{array}$} & \multicolumn{2}{c|}{$\begin{array}{c}\text { wl band-notched } \\
\text { slots }\end{array}$} \\
\cline { 3 - 6 } \multicolumn{2}{|c|}{} & S21 (dB) & RE(\%) & S21 (dB) & RE(\%) \\
\hline \multirow{2}{*}{$\begin{array}{c}\text { Type } \\
\text { A }\end{array}$} & $2.4 \mathrm{GHz}$ & -8 & 62 & -35 & 93 \\
\cline { 2 - 6 } & $5.2 \mathrm{GHz}$ & -17 & 78 & -33 & 87 \\
\hline $\begin{array}{c}\text { Type } \\
\text { B }\end{array}$ & $2.4 \mathrm{GHz}$ & -10 & 70 & -38 & 92 \\
\cline { 2 - 6 } & $5.2 \mathrm{GHz}$ & -13 & 85 & -26 & 90 \\
\hline \multirow{2}{*}{$\begin{array}{c}\text { Type } \\
\text { C }\end{array}$} & $2.4 \mathrm{GHz}$ & -24 & 83 & -24 & 84 \\
\cline { 2 - 6 } & $5.2 \mathrm{GHz}$ & -20 & 89 & -32 & 90 \\
\hline
\end{tabular}

To verify the characteristic of the band-notched slot, we change the length of the band-notched slot, $L_{1}$ and $L_{2}$. Fig. 6 shows that the notch frequency of slot can be controlled by changing the length of slot. In the same way, the bandnotched slots of type B suppress the surface current, reduce the mutual coupling and improve radiation efficiency. However, for the PIFAs of type C, which are orthogonally spaced structure, we find out the different tendency. The band-notched slot does a little affect on the lower frequency because the PIFAs of type $\mathrm{C}$ already have the separated surface current structure due to orthogonal position. The results are summarized in Table I.

To get a high diversity gain for the MIMO antenna system, the total antenna beam pattern of diversity system should be omni-directional. So, the null patterns of the azimuth plane should be compensated by a hybrid space-pattern diversity. Here, we deal with the switched diversity technique. Fig. 7 shows the measured and simulated beam pattern of the PIFA with the band-notched slots for type A and shows a good agreement. The former study has been shown that the antenna 
beam pattern of the finite ground plane is deeply characterized by the surface current distribution on the ground plane[8].

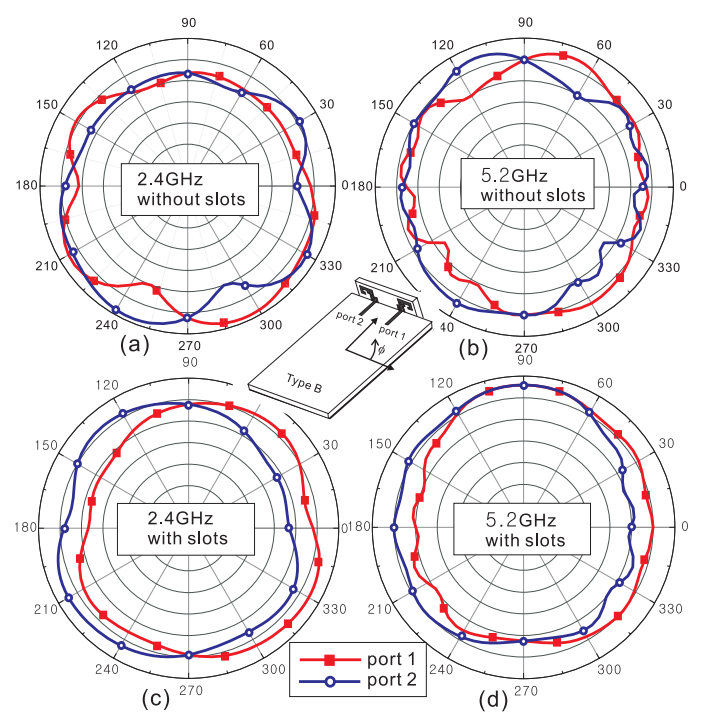

Fig. 9. Antenna gain patterns at the azimuth plane for the type B diversity system (a) at $2.4 \mathrm{GHz}$ without the band-notched slots (b) at $5.2 \mathrm{GHz}$ without the band-notched slots (c) at $2.4 \mathrm{GHz}$ with the bandnotched slots (d) at $5.2 \mathrm{GHz}$ with the band-notched slots

For the proposed antenna diversity system, the bandnotched slots separate the surface currents, which are caused by each antenna. Therefore, the beam pattern of two antennas is spontaneously divided into different directions. In Fig. 8, the complementary performance of the two antennas is depicted, proving that it is a hybrid space-pattern diversity system with omni-directional antennas. Fig. 8(a) shows that the radiation patterns of the PIFA without the band-notched slots have the null patterns. However, the radiation patterns of the PIFAs with the band-notched slots cover the null patterns as shown in Fig. 8(c). The similar results are obtained from other two types and are shown in Fig. 9 and 10, respectively.

\section{CONCLUSION}

In this paper, the internal dual band MIMO antenna with the dual band-notched slots for WLAN applications is proposed. By inserting the dual band-notched $\lambda / 4$ slots in the middle of the MIMO antennas, we create the high isolation and high radiation efficiency at each band. Moreover, by separating the surface current on the ground plane, the total radiation patterns are improved. These results surely can be contributed to the MIMO antennas with the small ground plane.

\section{ACKNOWLEDGEMENT}

This work was partially supported by the Brain Korea 21 project and Samsung Electro-Mechanics Co., Ltd.

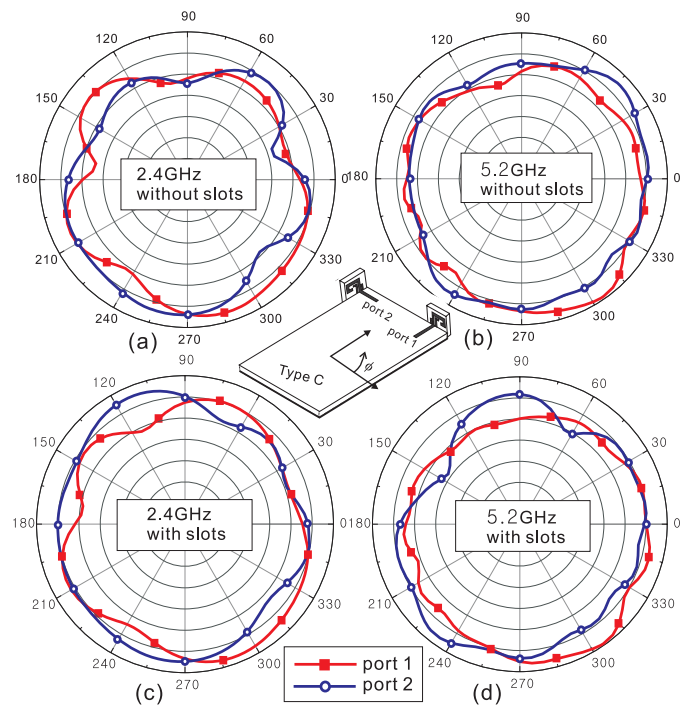

Fig. 10. Antenna gain patterns at the azimuth plane for the type C diversity system (a) at $2.4 \mathrm{GHz}$ without the band-notched slots (b) at $5.2 \mathrm{GHz}$ without the band-notched slots (c) at $2.4 \mathrm{GHz}$ with the bandnotched slots (d) at $5.2 \mathrm{GHz}$ with the band-notched slots

\section{REFERENCES}

[1] G. J. Foschini and M. J. Gans, “ On Limits of Wireless Communications in a Fading Environment when using Multiple Antennas," Wireless Personal Commun., vol. 6, no. 3. pp. 311-335, Mar. 1998.

[2] T. Svantesson and A. Ranheim, " Mutual Coupling Effects on the Capacity of Multi element Antenna Systems," in Proc. IEEE Int. Conf. Acoustics, Speech, and Signal Processing (ICASSP)' 01., vol. 4, May 2001, pp. 2485-2488

[3] Tzuenn-Yih Wu, Shyh-Tiring Fang, and, Kin-Lu Wong, “ A Printed Diversity Dual-band Monopole Antenna for WLAN Operation in the 2.4 and $5.2 \mathrm{GHz}$ Band," Microwave and Optical Technology Letters., vol. 36, no. 6, Mar 20, 2003

[4] Gaoming Chi, Binhong Li, and Dongsheng Qi, " Dual Band Printed Diversity Antenna for 2.4/5.2 GHz WLAN Application," Microwave and Optical Technology Letters., vol. 45, no. 6, June 202005

[5] Y. Gao, C.C. Chiau, X. Chen and C.G. Parini, " Modified PIFA and its array for MIMO terminals," IEE Proc.-Micorew. Antennas Propag., vol. 152, no. 4, Aug 2005

[6] M. Karaboikis. C. Soras, G. Tsachtsiris, and V. Makios, "Compact Dual-Printed Inverted-F Antenna Diversity Systems for Portable Wireless Devices," IEEE Antennas and Wireless Propagation Letters., vol. 3, 2004

[7] Kin-Lu Wong, Yen-Tu Chen, Saou-Wen Su and Yen-Liang Luo, " Diversity dual-band planar inverted-F antenna for WLAN operation," Microwave and Optical Technology Letters., vol. 38, no. 3, Aug. 5, 2005

[8] Nobuhiro Kuga, Kidenao Matsushima, and Naohisa Goto, " Plante-Loaded Folded Monopole antenna on a Ground-Plane Edge,” Electronics and Communications in Japa., Part I, vol. 8, no. 8, 2004 